\title{
Multicenter retrospective study of transcatheter arterial embolisation for life- threatening haemorrhage in patients with uncorrected bleeding diathesis
}

Stavros Spiliopoulos ${ }^{1,2^{*}}$ (D) Konstantinos Katsanos ${ }^{3}$, Ioannis Paraskevopoulos ${ }^{2}$, Martin Mariappan², Georgios Festas ${ }^{1}$, Panagiotis Kitrou ${ }^{3}$, Christos Papageorgiou ${ }^{3}$, Lazaros Reppas ${ }^{1}$, Konstantinos Palialexis ${ }^{1}$, Dimitrios Karnabatidis ${ }^{3}$ and Elias Brountzos ${ }^{1}$

\begin{abstract}
Background: We retrospectively investigated outcomes of emergency TAE for the management of life-threatening haemorrhage in patients with uncorrected bleeding diathesis.

Materials and methods: This multicenter, retrospective, study, was designed to investigate the safety and efficacy of percutaneous TAE for the management of life-threatening haemorrhage in patients with uncorrected bleeding disorder at the time of embolization. All consecutive patients with uncorrected coagulation who underwent TAE for the treatment of haemorrhage, between January 1st and December 31th 2019 in three European centers were included. Inclusion criteria were thrombocytopenia (platelet count $<50,000 / \mathrm{mL}$ ) and/or International Normalized Ratio (INR) $\geq 2.0$, and/or activated partial thromboplastin time (aPTT) $>45$ s, and/or a pre-existing underlying bloodclotting disorder such as factor VIII, Von Willebrand disease, hepatic cirrhosis with abnormal liver function tests. Primary outcome measures were technical success, rebleeding rate and clinical success. Secondary outcome measures included patients' 30-day survival rate, and procedure-related complications.

Results: In total, 134 patients underwent TAE for bleeding control. A subgroup of 17 patients with 18 procedures [11 female, mean age $70.5 \pm 15$ years] which represent $12.7 \%$ of the total number of patients, presented with pathological coagulation parameters at the time of TAE (haemophilia $n=3$, thrombocytopenia $n=1$, cirrhosis $n=5$, anticoagulants $n=7$, secondary to bleeding $n=1$ ) and were analyzed. Technical success was $100 \%$, as in all procedures the bleeding site was detected and successfully embolised. Clinical success was $100 \%$, as none of the patients died of bleeding during hospitalization, nor was surgically treated for bleeding relapse. Only one rebleeding case was noted (5.9\%) that was successfully treated with a second TAE. No procedure-related complications were noted. According to Kaplan-Meier analysis the estimated 30-day survival rate was $84.2 \%$.

(Continued on next page)
\end{abstract}

\footnotetext{
* Correspondence: stavspiliop@med.uoa.gr

²nd Department of Radiology, Interventional Radiology Unit, Medical

School, National and Kapodistrian University of Athens, "Attikon" University

Hospital, Athens, Greece

2Department of Clinical Radiology, Interventional Radiology Unit, Aberdeen

Royal Infirmary, NHS Grampian, Aberdeen AB25 2ZN, UK

Full list of author information is available at the end of the article
}

\section{Springer Open}

(c) The Author(s). 2020 Open Access This article is licensed under a Creative Commons Attribution 4.0 International License, which permits use, sharing, adaptation, distribution and reproduction in any medium or format, as long as you give appropriate credit to the original author(s) and the source, provide a link to the Creative Commons licence, and indicate if changes were made. The images or other third party material in this article are included in the article's Creative Commons licence, unless indicated otherwise in a credit line to the material. If material is not included in the article's Creative Commons licence and your intended use is not permitted by statutory regulation or exceeds the permitted use, you will need to obtain permission directly from the copyright holder. To view a copy of this licence, visit http://creativecommons.org/licenses/by/4.0/. 
(Continued from previous page)

Conclusion: TAE in selected patients with uncorrected bleeding diathesis should be considered as a suitable individualized management approach. Emergency TAE for life threatening haemorrhage in patients with coagulation cascade disorders should be used as an aid in realistic clinical decision making.

Keywords: Transcatheter arterial embolisation, Bleeding, Endovasdcular treatment, Coagulopathy, Prolonged clotting time

\section{Introduction}

Increasing experience and technological advances in embolic devices, coaxial catheter systems and microcatheters have established the role of percutaneous transcatheter arterial embolisation (TAE) in the modern treatment of haemorrhage (Rösch et al. 1972; Bookstein and Goldstein 1973; Bauer et al. 2004). TAE is today recommended in a variety of haemorrhagic conditions such as trauma, haemoptysis, tumors, etc. (Coccolini et al. 2017; Spiliopoulos et al. 2018; Chun et al. 2010; RevelMouroz et al. 2015; Chakraverty et al. 2012). Moreover, the continuously increasing population age, as well as the widespread use of more potent antithrombotic therapy has increased reports of severe spontaneous bleeding, while therapeutic anticoagulation at the time of bleeding, aggravates the severity of the event by triggering hemodynamic instability (Spiliopoulos et al. 2019). Additionally, underlying inherited or acquired coagulopathies, as well as acute traumatic coagulopathy and trauma induced coagulopathy, can exacerbate blood loss and delay treatment being independent predictors of mortality (Maegele 2019). Coagulopathies, either acquired (drug-induced, vitamin $\mathrm{K}$ deficiency, disseminated intravascular coagulation) or hereditary (hemophilia, von Willebrand disease), are not only correlated with an increased bleeding risk but have been also associated with clinical failure after transcatheter arterial embolization (Hur et al. 2014). Standard clinical practice in patients with clotting disorders includes correction of INR, aPPT and platelet values prior TAE, as this improves patient safety, increases clinical success and in several circumstances (mostly in spontaneous bleeding) incites haemostasis without further intervention (Spiliopoulos et al. 2018; Hur et al. 2014).

In 2012, the Society of Interventional Radiology (SIR), has issued a consensus document classifying endovascular procedures requiring sheath size up to $7 \mathrm{Fr}$, as medium risk procedures and recommended that should be performed after optimization of the coagulation profile to the following values: INR $<1.5$, aPTT $<1.5 \times$ control and platelet count $>50.000 / \mu \mathrm{L}$ (Patel et al. 2012).

In 2019, SIR released two updated consensus documents recommending arterial puncture $\leq 6 \mathrm{Fr}$ and embolotherapy for patients presenting with INR values between 2 and 3, and PLTs $<50,000 / \mu \mathrm{L}$, but this was based on an experts' consensus rather than solid scientific evidence (Davidson et al. 2019; Pearl et al. 2019). Moreover, available consensus documents do not include specific recommendations for the management of bleeding diathesis prior, during, and after TAE for haemorrhage control, which represents a separate clinical setting, requiring rapid decision making and intervention, especially for cases in which time to optimize coagulation profile is minimal (Chakraverty et al. 2012).

In every day clinical practice, although deranged clotting is not an absolute contraindication for TAE, many interventional radiologists refuse TAE for patients with uncorrected bleeding parameters, until INR value normalizes around 1.5, driven by the lack of evidence regarding patient safety, in combination with the documented insufficiency of specific embolic materials, such as metallic coils, to provoke blood clotting in the presence of haemostatic disorders (Patel et al. 2012; Shi et al. 2017). Data regarding the safety and efficacy of TAE in patients with severe, life-threatening haemorrhage and bleeding diathesis due to anticoagulation therapy or underlying coagulopathy, remain scarce as they are mainly based on subgroups of patients with coagulopathy included in single-center, retrospective series (Hur et al. 2014; Shi et al. 2017; Jaffe et al. 2015; Baron et al. 2013). The authors sought to investigate outcomes of emergency TAE, performed in three large European vascular centers, for the management of life-threatening hemorrhage in patients with uncorrected bleeding diathesis.

\section{Materials and methods}

This multicenter, retrospective, study, was designed to investigate the safety and efficacy of percutaneous TAE for the management of life-threatening haemorrhage in patients with uncorrected bleeding disorder at the time of embolization. Between January 1st and December 31th 2019 (one-year period), the electronic files all consecutive patients who underwent TAE in three European centers (A, P and A), for the treatment of haemorrhage, were searched and analysed. Institutional review board approval was not required for this retrospective study. A specific study informed consent was not necessary for inclusion in this retrospective study. A procedural written consent form has been obtained by all patients. 
Patients included in the analysis fulfilled the following criteria: (i) patients who underwent TAE due to haemorrhage within the predetermined 1-year period and (ii) uncorrected coagulation disorder at the time of embolization, defined as thrombocytopenia (platelet count $<50,000 / \mathrm{mL}$ ) and/or International Normalized Ratio (INR) $\geq 2.0$, and/or activated partial thromboplastin time $(\mathrm{aPTT})>45 \mathrm{~s}$, and/or a known underlying bloodclotting disorder such as factor VIII, Von Willebrand disease, hepatic cirrhosis with abnormal liver function tests (Hur et al. 2014; Patel et al. 2012). The study's flow chart is depicted in Fig. 1. In all participating centres, TAE was decided following a multidisciplinary team (MDT) decision involving the treating physician, the surgical team and the hematology team. The MDT organized persistent efforts to improve the coagulation profile prior, during and after embolization in all patients. The MDT decision to timely proceed to emergency embolization in patients with uncorrected bleeding diathesis was balanced and TAE was offered in cases in which patient survival was compromised by ongoing life-threatening bleeding leaving no available time for further improvement of the coagulation parameters. TAE was performed using standard interventional techniques according to international guidelines (Chakraverty et al. 2012). In brief, common femoral artery access was used and microcatheters were used (Progreat 2.7Fr; TERUMO or Masters 2.6Fr; ASHAHI INTECC, CO) for super-selective catheterization of the bleeding branches whenever necessary. The choice of embolic materials was case-sensitive and decided by the operator according to his experience, the embolization goal and the medical centre's device availability.

\section{Statistics}

Continuous data regarding rebleeding rate were evaluated with the Kolmogorov-Smirnov goodness-of-fit test to determine whether they were originating from normal distributions and the Mann-Whitney test was performed for the comparison of rebleeding rate between the two cohorts of deranged and normal coagulation profiles, as variables did not pass the normality test. Patients' 30-day survival was estimated using Kaplan-Meier analysis. Statistical analysis was performed with SPSS statistical software (version 25, IBM).

\section{Endpoints and definitions}

The primary outcome measures were technical success, defined as occlusion of bleeding site(s) with no signs of bleeding at completion DSA, recurrent haemorrhage rate defined as relapse of bleeding at the same site following successful TAE, re-intervention rate due to recurrent haemorrhage and clinical success defined as the absence of bleeding related death during hospitalization. Secondary outcome measures were patients' 30-day survival rate, rebleeding rate compared to that of patients

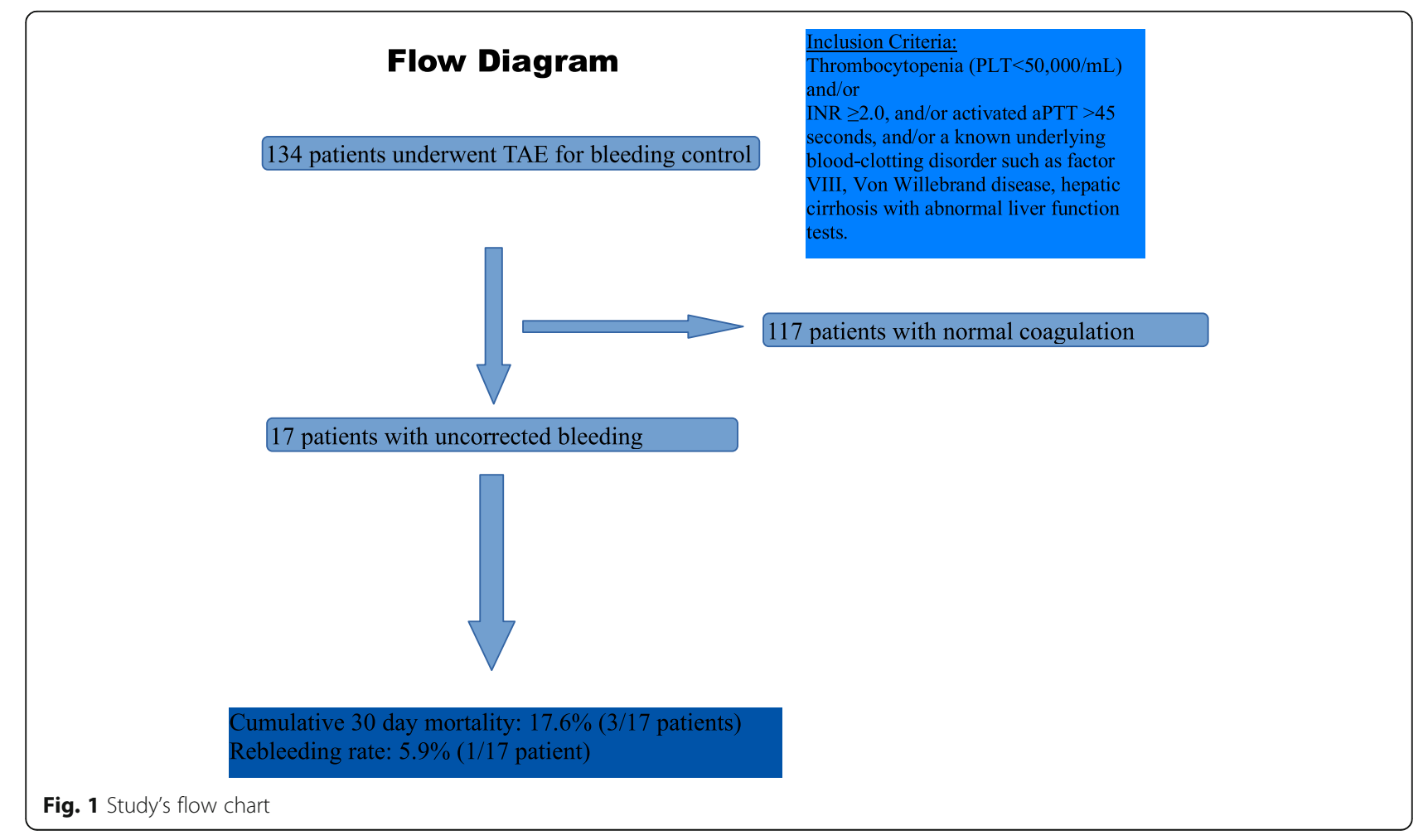


without deranged coagulation at the time of embolization, and procedure-related complications, classified according to the CIRSE 6-grade classification system (Filippiadis et al. 2017). All primary and secondary outcomes were statistically analysed on a per patient basis.

\section{Results}

In total, 134 patients underwent TAE for bleeding control within the study's one-year time period and $12.7 \%$ [17 patients; 11 female $(64.7 \%)$ ] with a mean age $70.5 \pm 15$ years (range: $23-87$ ), presented with pathological coagulation parameters indicating increased bleeding diathesis at the time of TAE and were enrolled in the study. All 17 patients underwent computed tomography angiography (CTA) which was positive for active bleeding prior the procedure. In total $18 \mathrm{TAE}$ procedures were performed and analysed. In one patient underlying coagulopathy (congenital haemophilia) was not known at the time of embolization and all other bleeding parameters were within normal range. INR $\geq 2$ was noted in seven patients (two with concomitant prolonged aPTT $>45$ ). Prolonged aPTT $>45$ was observed in five patients. Underlying Coagulopathy was recorded in seven patients and included three cases of haemophilia (two cases of congenital Von Willebrand disease and one case of acquired factor VIII deficiency), one case of thrombocytopenia (PLTs $29 \mathrm{~K} / \mu \mathrm{L}$ ) and five cases of cirrhosis with severe total protein and albumin deficit (three cases of decompensated cirrhosis). In three patients with underlying coagulopathy, INR values $\geq 1.7$ were recorded (two with cirrhosis and one with acquired factor VIII deficiency). Patients' baseline demographical and clinical-laboratory data, as well as procedural details are analytically presented in Table 1 .

In most of the cases liquid embolic material was preferred such as N-butyl cyanoacrylate (Gluebran ${ }^{\circ}$, GEM $\mathrm{SRL}$ ) in three cases; Onyx (Medtronic) in two cases and gelfoam (Gelfoam; Pfizer or Embocube $2.5 \mathrm{~mm}$; MERIT Medical) in six cases (11/18 procedures; $61.1 \%)$, either alone $(3 / 18$ procedures; $16.6 \%)$ or in combination with other embolic materials such as microparticles (Embozene microspheres; CELONOVA or Embosphere ${ }^{\circ} \mathrm{Mi}-$ crospheres; MERIT Medical) or microcoils (pushable Nestercoils; COOK, or CONCERTO ${ }^{\mathrm{mm}}$ Helix detachable microcoils; Medtronic) or both (Table 1). In four procedures only microcoils were used $(4 / 18 ; 22.2 \%)$.

Technical success was $100 \%$, as in all procedures the bleeding site was detected and embolized, without angiographical signs of haemorrhage at final DSA. Clinical success was $100 \%$, as none of the patients died of bleeding during hospitalization, nor was surgically treated for bleeding relapse. In all 18 procedures $6 \mathrm{Fr}$ sheaths were used and a closure device was deployed to achieve haemostasis in all cases (seven Angio-Seal ${ }^{\mathrm{Tw}}$; TERUMO Europe, Belgium; ten Starclose $\mathrm{SE}^{\mathrm{rm}}$ and one Perclose ProGlide $^{\mathrm{in}}$; ABBOTT, USA). No access-site bleeding complications were noted, apart from access-related soft, small groin haematomas. Rebleeding occurred in one patient (rebleeding rate 5.9\%; $1 / 17$ cases). The single rebleeding episode occurred in an 87-year old female patient with acquired factor VIII deficiency (attributed to pneumonia), heparin therapy for venous thrombosis (enoxaparin $40 \mathrm{mg}$ twice daily), and spontaneous active bleeding from a right deep femoral artery DFA branch causing enlarging thigh haematoma. Factor VIII was not readily available at the hospital and was awaited. Hemoglobin $(\mathrm{Hb})$ dropped from $8.5 \mathrm{~g} / \mathrm{dL}$ at arrival to $7.5 \mathrm{~g} / \mathrm{dL}$ in $2 \mathrm{~h}$ and further decreased to $5.1 \mathrm{~g} / \mathrm{dL}$ at the time of the first embolization, despite transfusions (2 bags of plasma 2 units of RBCs and transamine administration). The patient was unstable (systolic blood pressure (SBP): $65 \mathrm{mmHg}$ under inotropic therapy] INR was 1.8 and aPTT was 164.1 at the time of the first technically successful embolization using gel foam (Fig. 2a-b). The patient was immediately stabilized (SBP $100 \mathrm{mmHg}$ without inotropic support) and $\mathrm{Hb}$ increased to $7.8 \mathrm{~g} / \mathrm{dL}$. However, after $12 \mathrm{~h} \mathrm{Hb}$ dropped to $6.5 \mathrm{~g} / \mathrm{dL}$ and aPTT was again indefinable. The patient was now haemodynamically stable. CTA detected rebleeding at the same location. DSA confirmed bleeding from the previously embolized DFA branch (Fig. 2c) and reintervention using further foam and coiling of the origin of the DFA was successfully performed (Fig. 2d). The patient received factor VIII approximately $8 \mathrm{~h}$ after the second embolization and no further bleeding was noted. She died after 17 days in ICU due to respiratory insufficiency caused by pneumonia. Rebleeding rate was similar compared to the cohort of patients embolized during the same time period $[5.9 \%$ (1/17 cases) versus $4.3 \%$ (5/117 cases), $p=0.38$; respectively]. No procedure-related complications were noted. In three cases (3/18 procedures; $16.6 \%)$ the performing physician decided to remove the arterial sheath at approximately 24 and $72 \mathrm{~h}$ following the procedureIn all cases a closure device was used for haemostasis, without any sequalae. The day following TAE, normalization of the coagulation profile (INR $\leq 1.7$ and $\mathrm{aPTT}<45$ and platelet count $>50,000 / \mathrm{mL}$ ) was noted in $7 / 12$ cases (58.3\%) with previously abnormal INR and/or aPTT and/or PLT count.

In total, three deaths were recorded during hospitalization and the cumulative 30-day mortality rate was $17.6 \%$ (3/17 cases). According to Kaplan-Meier analysis the estimated 30-day survival rate was $84.2 \%$ (Fig. 3). Death rate directly related to bleeding was zero, as two patients died of pneumonia in ICU 17 days and 20 days after TAE (patients \#5 and \#11 in Table 1 ) and one due to cholangitis 12 days after TAE (patient \#16 in Table 1). 
Table 1 Demographical data, coagulation data and procedural details

\begin{tabular}{|c|c|c|c|c|c|c|c|c|c|c|}
\hline $\mathrm{Pt}$ & Gender & $\begin{array}{l}\text { Age } \\
\text { (years) }\end{array}$ & $\begin{array}{l}\text { Underlying } \\
\text { pathology/ } \\
\text { anticoagulation }\end{array}$ & $\begin{array}{l}\text { Coagulation } \\
\text { profile }\end{array}$ & Cause & Bleeding vessel & $\begin{array}{l}\text { Embolization } \\
\text { material }\end{array}$ & $\begin{array}{l}\text { Sheath } \\
\text { size/ } \\
\text { removed }\end{array}$ & $\begin{array}{l}\text { Closure } \\
\text { device }\end{array}$ & Rebleeding \\
\hline 1 & Female & 75 & Warfarin & $\begin{array}{l}\text { INR 2.2; PT } \\
\text { 19.2; aPTT: } \\
\text { 27.8; PLT: } \\
258 \mathrm{~K} / \mu \mathrm{L}\end{array}$ & Spontaneous & $\begin{array}{l}\text { Lt Subclavian } \\
\text { artery branch }\end{array}$ & $\begin{array}{l}\text { Pushable } \\
\text { microcoils }(\times 2) \\
\text { and } 300-500 \mu \mathrm{m} \\
\text { microparticles }\end{array}$ & $\begin{array}{l}6 \mathrm{Fr} / \\
\text { same day }\end{array}$ & Angioseal & No \\
\hline 2 & Male & 83 & Warfarin & $\begin{array}{l}\text { INR 2.14; PT } \\
\text { 20.3; aPTT: } \\
\text { 37.4; PLT: } \\
\text { 184 K } \mu \mathrm{LL}\end{array}$ & Trauma & $\begin{array}{l}\text { Right renal } \\
\text { artery branch }\end{array}$ & $\begin{array}{l}\text { Gelfoam and } \\
\text { pushable } \\
\text { microcoils (×3) }\end{array}$ & $\begin{array}{l}6 \mathrm{Fr} / \\
\text { same day }\end{array}$ & Perclose & No \\
\hline 3 & Female & 70 & Warfarin & $\begin{array}{l}\text { INR 4.3; PT } \\
\text { 41.1; aPTT: } \\
\text { 33.1; PLT: } \\
141 \mathrm{~K} / \mu \mathrm{L}\end{array}$ & Spontaneous & $\begin{array}{l}\text { Right inferior } \\
\text { epigastric artery }\end{array}$ & Glue 1:3 & $\begin{array}{l}6 \mathrm{Fr} / \\
\text { same day }\end{array}$ & Starclose & No \\
\hline 4 & Female & 82 & $\begin{array}{l}\text { Giant AML }(16 \mathrm{~cm}) \\
\text { rupture }\end{array}$ & $\begin{array}{l}\text { INR 3.5; PT } \\
\text { 20.3; aPTT: } \\
\text { 37.4; PLT: } \\
\text { 184 K/ } \mu \mathrm{L}\end{array}$ & Trauma & $\begin{array}{l}\text { Left renal artery } \\
\text { branches }\end{array}$ & $\begin{array}{l}\text { Microspheres } \\
300-500 \text { and } \\
500-700 \text { and } \\
\text { microcoils }(\times 3)\end{array}$ & $6 \mathrm{Fr} / 24 \mathrm{~h}$ & Starclose & No \\
\hline 5 & Female & 87 & $\begin{array}{l}\text { Acquired factor VIII } \\
\text { deficiency/ } \\
\text { LMWH } 40 \text { mg twice } \\
\text { daily }\end{array}$ & $\begin{array}{l}\text { INR 1.8; PT } \\
\text { 21.5; aPTT: } \\
\text { 164.1; PLT: } \\
483 \mathrm{~K} / \mu \mathrm{L}\end{array}$ & Spontaneous & $\begin{array}{l}\text { Right deep } \\
\text { femoral artery } \\
\text { branches }\end{array}$ & Gelfoam & $\begin{array}{l}6 \mathrm{Fr} / \\
\text { same day }\end{array}$ & Starclose & $\begin{array}{l}\text { Yes; } \\
\text { successfully } \\
\text { treated } \\
\text { with } 3 \\
\text { microcoils } \\
\text { and } \\
\text { gelfoam }\end{array}$ \\
\hline 6 & Female & 23 & $\begin{array}{l}\text { Congenital } \\
\text { hemophilia }\end{array}$ & $\begin{array}{l}\text { INR 1.37; PT } \\
\text { 14.3; aPTT: } \\
\text { 30.5; PLT: } \\
141 \mathrm{~K} / \mu \mathrm{L}\end{array}$ & $\begin{array}{l}\text { latrogenic: } \\
\text { post renal } \\
\text { biopsy }\end{array}$ & $\begin{array}{l}\text { Right renal } \\
\text { artery branch }\end{array}$ & $\begin{array}{l}\text { Glue }(1: 1) \text { and } \\
\text { pushable } \\
\text { microcoil }(\times 1)\end{array}$ & $\begin{array}{l}6 \mathrm{Fr} / \\
\text { same day }\end{array}$ & Angioseal & No \\
\hline 7 & Female & 74 & $\begin{array}{l}\text { Decompensated } \\
\text { cirrhosis: Alk phos } 50 \\
\mathrm{U} / \mathrm{L} \text {; Albumin } 12 \mathrm{~g} / \mathrm{L} \text {; } \\
\text { total proteins } 21 \mathrm{~g} / \mathrm{L} \text {, } \\
\text { Bilirubin } 22 \mathrm{umol} / \mathrm{L}\end{array}$ & $\begin{array}{l}\text { INR 1.0; PT } \\
\text { 12.1; aPTT: } \\
\text { 29.8; PLT: } 55 \\
\text { K/LL }\end{array}$ & $\begin{array}{l}\text { latrogenic: } \\
\text { post-liver } \\
\text { biopsy }\end{array}$ & $\begin{array}{l}\text { Right hepatic } \\
\text { artery }\end{array}$ & $\begin{array}{l}\text { Microparticles } \\
500-700 \mu \mathrm{m} \text { and } \\
\text { gelfoam }\end{array}$ & $\begin{array}{l}6 \mathrm{Fr} / \\
\text { same day }\end{array}$ & Starclose & No \\
\hline 8 & Female & 72 & $\begin{array}{l}\text { Decompensated } \\
\text { cirrhosis/gastric } \\
\text { varices: Alk phos } 67 \\
\text { U/L; Albumin } 19 \mathrm{~g} / \mathrm{L} \text {; } \\
\text { total proteins } 31 \mathrm{~g} / \mathrm{L} \text {, } \\
\text { Bilirubin } 54 \text { umol/L }\end{array}$ & $\begin{array}{l}\text { INR 4.5; PT } \\
\text { 46; aPT: } \\
\text { 124.2; PLT: } \\
348 \mathrm{~K} / \mu \mathrm{L}\end{array}$ & Spontaneous & $\begin{array}{l}\text { Left hepatic } \\
\text { artery }\end{array}$ & $\begin{array}{l}\text { Microparticles } \\
300-500 \mu \mathrm{m} \\
\text { and pushable } \\
\text { microcoils }(\times 3)\end{array}$ & $\begin{array}{l}6 \mathrm{Fr} / \\
\text { same day }\end{array}$ & Starclose & No \\
\hline 9 & Female & 68 & $\begin{array}{l}\text { LMWH } 60 \text { mg once } \\
\text { daily }\end{array}$ & 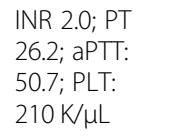 & Trauma & $\begin{array}{l}\text { Right internal } \\
\text { iliac artery }\end{array}$ & $\begin{array}{l}\text { Pushable } \\
\text { microcoils (x9) }\end{array}$ & $\begin{array}{l}6 \mathrm{Fr} / \\
\text { same day }\end{array}$ & Starclose & No \\
\hline 10 & Female & 75 & $\begin{array}{l}\text { LMWH } 40 \text { mg twice } \\
\text { daily }\end{array}$ & $\begin{array}{l}\text { INR 1.2; PT } \\
\text { 17.5; aPTT: } \\
\text { 121.3; PLT: } \\
176 \mathrm{~K} / \mu \mathrm{L}\end{array}$ & Spontaneous & DFA branch & $\begin{array}{l}\text { Pushable } \\
\text { microcoils }(\times 2)\end{array}$ & $\begin{array}{l}6 \mathrm{Fr} / \\
\text { same day }\end{array}$ & Starclose & No \\
\hline 11 & Male & 64 & Warfarin & $\begin{array}{l}\text { INR 4.7; PT: } \\
\text { 74.1; aPTT: } \\
\text { 48.3; PLT: } \\
348 \mathrm{~K} / \mu \mathrm{L}\end{array}$ & Spontaneous & SMA branch & $\begin{array}{l}\text { Pushable } \\
\text { microcoils ( } \times 7)\end{array}$ & $6 \mathrm{Fr} / 24 \mathrm{~h}$ & Angioseal & No \\
\hline 12 & Female & 86 & $\begin{array}{l}\text { LMWH } 40 \text { mg twice } \\
\text { daily }\end{array}$ & 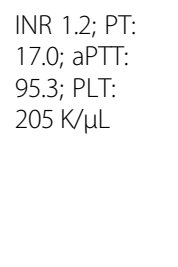 & Spontaneous & $\begin{array}{l}\text { Left inferior } \\
\text { epigastric artery } \\
\text { and left deep } \\
\text { femoral artery } \\
\text { branches }\end{array}$ & $\begin{array}{l}\text { Onyx Ethylene } \\
\text { vinyl alcohol } \\
\text { copolymer } \\
(\text { EVOH), } \\
\text { Microparticles } \\
300-500 \mu \mathrm{m} \text { and } \\
\text { pushable micro- } \\
\text { coils }(\times 5)\end{array}$ & $\begin{array}{l}6 \mathrm{Fr} / \\
\text { same day }\end{array}$ & Starclose & No \\
\hline 13 & Male & 59 & Thrombocytopenia & $\begin{array}{l}\text { INR 1.2; PT: } \\
\text { 12.7; аРTT: 36; }\end{array}$ & Spontaneous & $\begin{array}{l}\text { Left 3rd Lumbar } \\
\text { artery }\end{array}$ & $\begin{array}{l}\text { Onyx Ethylene } \\
\text { vinyl alcohol }\end{array}$ & $6 \mathrm{Fr} / 72 \mathrm{~h}$ & Angioseal & No \\
\hline
\end{tabular}


Table 1 Demographical data, coagulation data and procedural details (Continued)

\begin{tabular}{|c|c|c|c|c|c|c|c|c|c|c|}
\hline $\mathrm{Pt}$ & Gender & $\begin{array}{l}\text { Age } \\
\text { (years) }\end{array}$ & $\begin{array}{l}\text { Underlying } \\
\text { pathology/ } \\
\text { anticoagulation }\end{array}$ & $\begin{array}{l}\text { Coagulation } \\
\text { profile }\end{array}$ & Cause & Bleeding vessel & $\begin{array}{l}\text { Embolization } \\
\text { material }\end{array}$ & $\begin{array}{l}\text { Sheath } \\
\text { size/ } \\
\text { removed }\end{array}$ & $\begin{array}{l}\text { Closure } \\
\text { device }\end{array}$ & Rebleeding \\
\hline & & & & PLT: $29 \mathrm{~K} / \mu \mathrm{L}_{;}$ & & & $\begin{array}{l}\text { copolymer } \\
\text { (EVOH) }\end{array}$ & & & \\
\hline 14 & Male & 65 & $\begin{array}{l}\text { Congenital } \\
\text { hemophilia }\end{array}$ & $\begin{array}{l}\text { INR 1.1; PT } \\
\text { 12.0; aPTT: } \\
\text { 29.3; PLT: } \\
152 \mathrm{~K} / \mu \mathrm{L}\end{array}$ & $\begin{array}{l}\text { latrogenic: } \\
\text { post-arterial } \\
\text { access }\end{array}$ & $\begin{array}{l}\text { Right deep } \\
\text { femoral artery } \\
\text { pseudoaneurysm }\end{array}$ & $\begin{array}{l}\text { Glue 1:1 and } \\
\text { pushable } \\
\text { microcoil }(\times 1)\end{array}$ & $\begin{array}{l}6 \mathrm{Fr} / \\
\text { same day }\end{array}$ & Angioseal & No \\
\hline 15 & Male & 66 & $\begin{array}{l}\text { Cirrhosis; Alk phos } 67 \\
\mathrm{U} / \mathrm{L} \text {; Albumin } 21 \mathrm{~g} / \mathrm{L} ; \\
\text { total proteins } 63 \mathrm{~g} / \mathrm{L} \text {, } \\
\text { Bilirubin } 228 \mathrm{umol} / \mathrm{L}\end{array}$ & $\begin{array}{l}\text { INR 1.7; PT: } \\
\text { 20; aPTT: } 39.4 ; \\
\text { PLT: } 59 \mathrm{~K} / \mu \mathrm{L}\end{array}$ & Spontaneous & $\begin{array}{l}\text { Left inferior } \\
\text { epigastric artery }\end{array}$ & $\begin{array}{l}\text { Gelfoam and } \\
\text { pushable } \\
\text { microcoils }(\times 2)\end{array}$ & $\begin{array}{l}6 \mathrm{Fr} / \\
\text { same day }\end{array}$ & Starclose & No \\
\hline 16 & Male & 82 & $\begin{array}{l}\text { Decompensated } \\
\text { cirrhosis/ jaundice: } \\
\text { Alk phos } 227 \mathrm{U} / \mathrm{L} ; \\
\text { Albumin } 17 \mathrm{~g} / \mathrm{L} ; \text { Total } \\
\text { protein } 44 \mathrm{~g} / \mathrm{L} ; \mathrm{AAT} \\
127 \mathrm{U} / \mathrm{L} ; \mathrm{GGT} 211 \mathrm{U} / \mathrm{L} ; \\
\text { Bilirubin } 217 \mathrm{umol} / \mathrm{L}\end{array}$ & $\begin{array}{l}\text { INR 1.0; PT: } \\
\text { 10.6; aPTT: } \\
\text { 32.9; PLT: } \\
185 \mathrm{~K} / \mu \mathrm{L}\end{array}$ & Spontaneous & $\begin{array}{l}\text { Left gastric } \\
\text { artery }\end{array}$ & $\begin{array}{l}\text { Pushable } \\
\text { microcoils }(x 4)\end{array}$ & $\begin{array}{l}6 \mathrm{Fr} / \\
\text { same day }\end{array}$ & Angioseal & No \\
\hline 17 & Female & 69 & $\begin{array}{l}\text { Cirrhosis:: Alk phos } \\
114 \text { U/L; Albumin } 31 \\
\text { g/L; Total protein } 54 \\
\text { g/L; AAT 44 U/L; GGT } \\
114 \text { U/L; Bilirubin } \\
\text { 24umol/L }\end{array}$ & $\begin{array}{l}\text { INR 1.7; PT: } \\
\text { 19.3; aPTT: } \\
\text { 26.7; PLT: } \\
166 \mathrm{~K} \mu \mathrm{L}\end{array}$ & Spontaneous & $\begin{array}{l}\text { Right hepatic } \\
\text { artery }\end{array}$ & $\begin{array}{l}\text { Microparticles } \\
300-500 \mu \mathrm{m} \text {, de- } \\
\text { tachable micro- } \\
\text { coil }(\times 1) \text {, } \\
\text { gelfoam }\end{array}$ & $\begin{array}{l}6 \mathrm{Fr} / \\
\text { same day }\end{array}$ & Angioseal & No \\
\hline
\end{tabular}

\section{Glue used: N-butyl-2 cyanoacrylate (NBCA)}

Microparticles used: Embozene microspheres; CELONOVA and Embosphere ${ }^{\circledast}$ Microspheres; MERIT Medical

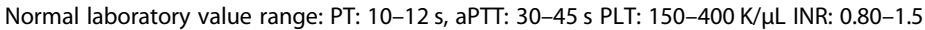

Compication rate: $5,9 \%(1 / 17)$

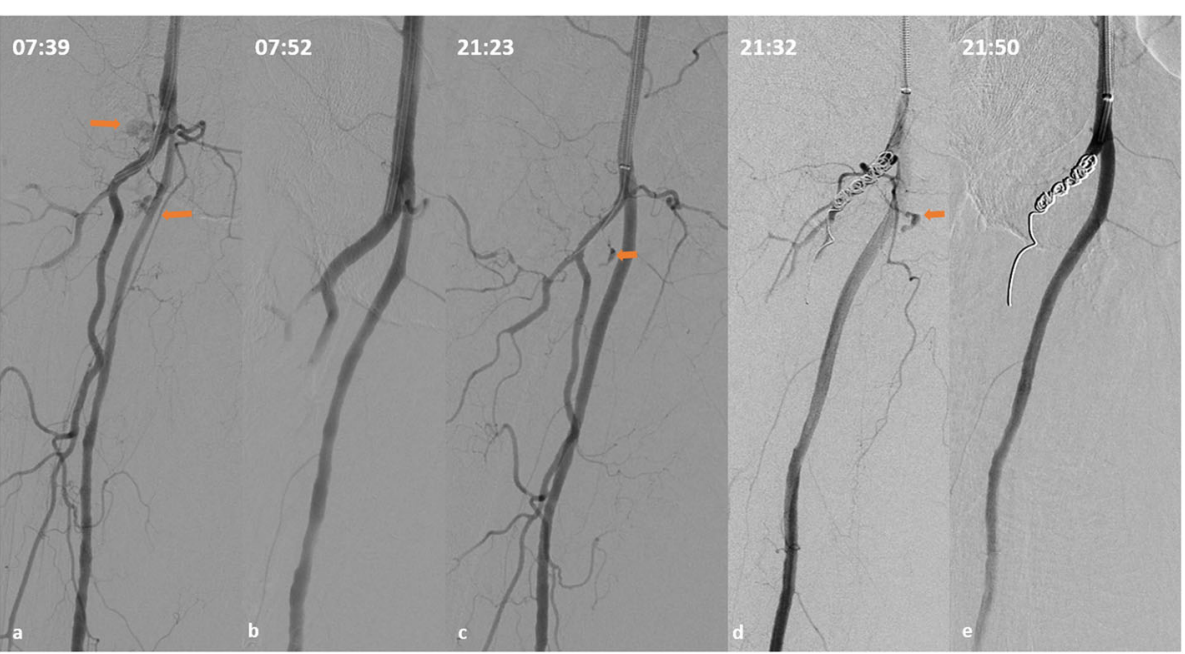

Fig. 2 The single case of rebleeding. An 87-year old female patient with acquired factor VIII deficit, aPTT 164.1; INR 1.8 and spontaneous bleeding of a right deep femoral artery branch, causing significant $\mathrm{Ht} / \mathrm{Hb}$ decrease and hemodynamic instability. a Selective DSA demonstrating two sites of active extravasation from branches of the DFA. b Final DSA following gel foam embolization. c Selective DSA approx. $13 \mathrm{~h}$ after first embolization demonstrating rebleeding from one of the previously embolized bleeding sites (arrow). aPTT is now prolonged. $\mathbf{d}$ Persisting active bleeding through the coils, approx 5 min after their deployment. e Final DSA demonstrating successful hemostasis following further embolization with gel foam after placing the microcatheter within the coils 


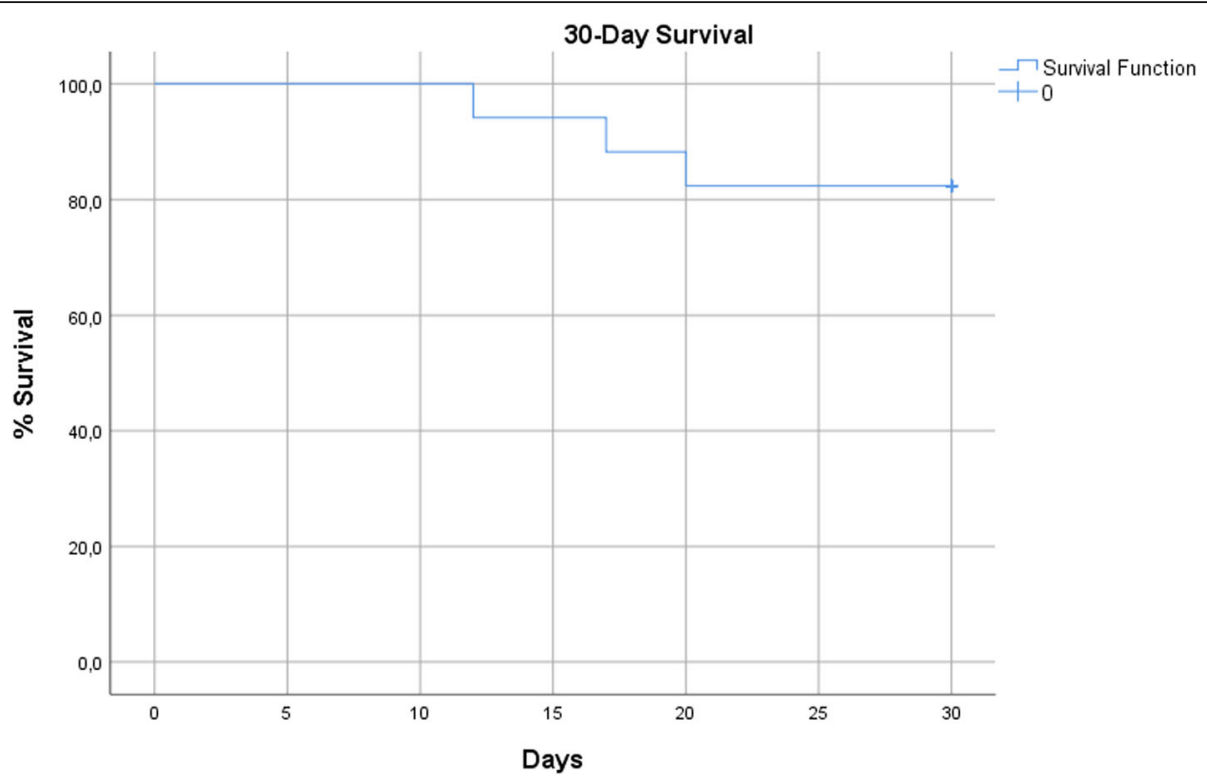

Fig. 3 Kaplan-Meier plot of 30-day survival rate

\section{Discussion}

The annual incidence of TAE procedures performed in the three participating tertiary European vascular centres for the management of severe haemorrhage in patients with uncorrected bleeding diathesis, was surprisingly high (12.7\%; approximately one out of ten patients who underwent TAE in 2019). The results of this retrospective, real-life, study, demonstrated that TAE in the specific cases was safe and effective and could increase the therapeutic window in carefully selected patients. Specifically, technical success was $100 \%$ and only one case of rebleeding/repeat TAE was recorded that was successfully treated with further TAE. The clinical safety and effectiveness of TAE has been widely reported in the literature, establishing its role in bleeding control (Spiliopoulos et al. 2019; Hur et al. 2014; Melloul et al. 2015; Padia et al. 2020). However, uncorrected coagulopathy related to anticoagulation, haemophilia, cirrhosis or severe trauma is a relative contraindication for TAE, as it has been previously reported as a significant negative predictor for recurrent bleeding, in-hospital mortality and procedure-related complication rates, up to 3.5 times more likely to result in clinical failure (Hur et al. 2014; Valek and Husty 2013; Angle et al. 2010; Loffroy et al. 2010; Lopera 2010). Loffroy et al., have reported that patients with impaired coagulation demonstrate three times higher risk for rebleeding following successful TAE and ten times higher bleeding-related risk of death compared to patients with normal coagulation profile. Nevertheless, the authors highlighted the fact that these patients should not be refused emergency TAE while efforts to correct coagulation before, during, and after embolization, are of most importance for definite clinical success (Loffroy et al. 2010). This study reports similar technical success and rebleeding rates between patients with severe bleeding diathesis and those with normal coagulation profile at the time of embolization. Moreover, no procedure-related complications were noted, while the 30-day survival rate was considered extremely satisfactory, given the advanced age and multiple comorbidities noted in the specific cohort.

The authors speculate that the above-mentioned outcomes could be attributed to several factors, among which are the long-standing local experience and expertise in the multi-disciplinary management of haemorrhage, that includes optimal periprocedural coagulation improvement, as well as specific considerations regarding the timing and technique of TAE. Notably, efforts to improve the coagulation profile prior, during and after embolization were made in all patients and in $58 \%$ of the cases, abnormal INR, aPTT and PLT values were normalized within $24 \mathrm{~h}$, a fact that certainly contributed to the minimal rebleeding rate and overall clinical success. The decision to timely proceed to emergency embolization in patients with uncorrected bleeding diathesis was balanced between the risk of technical failure/procedure-related complications and patient survival, which was severely compromised by ongoing bleeding causing hemodynamic instability, leaving no time for further improvement of the coagulation parameters. Such emergency multidisciplinary clinical decisions are currently supported by international experts' consensus (Patel et al. 2012). Of note, time to 
embolization has been correlated with better clinical outcomes and waiting for a long time period in order to normalize clotting time is not a good option in cases of large-volume bleeding causing hemodynamic instability (Chakraverty et al. 2012).

In this study, the choice of embolic material was heterogeneous and highly individualized, a fact that should be expected in a retrospective analysis. It is well-known that correct choice of the embolization agents is essential. However, in patients with impaired coagulation, the choice of embolic agents is even more crucial and should be based on unique particularities. The use of metallic coils has been correlated with ineffective haemostasis, as their mechanism of action is based on the organism's intrinsic ability to form clot. This is less likely to occur when the coagulation cascade has been impaired. In this series, blood flow through the coils has been noted even $5 \mathrm{~min}$ after coil deployment in a patient with factor VIII deficiency and an undefinable aPTT value (Fig. 1). The same applies for gelfoam as its mechanism of action also involves mechanical obstruction, blood flow reduction and therefore thrombus formation. However, dense coil packing or a combination of dense coiling with gelfoam could provoke complete arterial blockage and successful hemostasis (Fig. 1). Solely dense coil embolization was successfully performed in four cases, without any bleeding recurrence. Although, not commonly preferred, successful coil-only embolization has been previously reported for patients with significantly prolonged INR (>3) (Ramaswamy 2014). Choosing to use only coils, is usually based on the safety provided by coiling in order to avoid nontargeted embolization and severe ischemic complications, such as in cases in which selective catheterization is not possible or for lesions close to arterial origins and bifurcations (Fig. 1), where glue or particles could easily migrate. It is important to understand that correct dense coil packing can be efficient even in cases of deranged clotting, especially if prompt normalization of the coagulation profile is awaited shortly after TAE. Microparticles, also act as blood-flow blockers, but their mechanism of action also involves an immediate inflammatory reaction of the arterial wall, focal angionecrosis and eventual vessel fibrosis and theoretically should be less influenced by deranged coagulation. In this study, microparticles were successfully used but only in combination with microcoils, and generally various combination of embolic agents was used in most cases (in order to enhance the occlusive effect, as previously described (Aina et al. 2001). On the other hand, n-butyl cyanoacrylate glue and ethylene vinyl alcohol copolymer $\left(O n y x^{\circ}\right)$ have been proposed as the most effective embolic agents in patients with bleeding disorders, as polymerization (glue) and solidification (Onyx) abruptly occludes the target vessel independently from the coagulation process (Shi et al. 2017; Toyoda et al. 1996; Né et al. 2018).

In the single case of rebleeding, gel foam was used a spontaneous bleeding originating from a DFA branch, in a patient with acquired factor VIII deficit and hemodynamic instability. Re-embolization using a combination of microcoils and gel foam was successfully performed, and bleeding control was achieved following concomitant factor VIII administration, which was not available at the time of initial embolization. Despite bleeding relapse, the first TAE procedure, contributed in stabilising the patient hemodynamically. The second hemorrhagic event occurred after $12 \mathrm{~h}$. Subsequent second successful TAE in combination with factor VIII administration resulted in successful haemostasis.

Another important issue is access-site haemostasis and related complications. Manual compression in patients with prolonged coagulation time can be extremely long-lasting, tedious and ineffective. A closure device was used in all patients and no access-site complications were noted. In two cases the sheath was removed the next day and in one case after approximately $72 \mathrm{~h}$. Choosing not to remove the sheath immediately after the procedure is always a choice if abnormal coagulation parameters are expected to normalize or significantly improve over time. Smaller $4 \mathrm{Fr}$ or $5 \mathrm{Fr}$ sheaths would be an option in these high bleeding risk cohort, but hemostasis should be performed using manual compression, as available closure devices use $6 \mathrm{Fr}$ sheaths. The authors preferred the use of a closure device over manual compression for smaller access diameters in the specific cohort, although this is not supported by evidence.

Limitations of this study include the retrospective design which could generate a selection bias, while some cases and data could have been missed. Moreover, cases that were discussed by the MDT for possible TAE, but finally underwent endoscopy or surgery, could not be identified. Additionally, this was not designed to be a comparative study with a control group and comparative data of rebleeding rate with the group of patients demonstrating normal coagulation profile could be confounded by significant heterogeneity. Notably, additional conservative treatments given to patients (for example cryoprecipitate) may have increased procedural and clinical success, but study design did not allow for the assessment of the contribution of such factors to the study outcome measures. Finally, the relatively small number of patients included, limits the validity of outcomes. 


\section{Conclusions}

This study provides valuable data supporting the option of life-saving TAE for haemorrhage control in selected patients with uncorrected bleeding diathesis, in order to avoid unnecessary delays while trying to correct coagulation parameters that either cannot be corrected or do not require correction for emergency TAE.

\section{Abbreviations}

aPTT: Activated Partial Thromboplastin Time; DFA: Deep Femoral Artery; DSA: Digital Subtraction Angiography; Hb: Hemoglobin; Ht: Hematocrit; ICU: Intensive Care Unit; INR: International Normalized Ratio; PLT: Platelets; PT: Prothrombin time; TAE: Transcatheter Arterial Embolisation

\section{Acknowledgements}

None.

\section{Authors' contributions}

Guarantor of integrity of the entire study: E. Brountzos, Study concept and design: S. Spiliopoulos, E. Brountzos, Literature research: K. Katsanos, S. Spiliopoulos, P. Kitrou, L. Reppas, K. Palialexis, D. Karnabatidis, I. Paraskevopoulos, M. Mariappan, G. Festas, C. Papageorgiou, D. Karnabatidis. Statistical analysis: K. Katsanos, S. Spiliopoulos, G. Festas. Manuscript preparation: M. Mariappan, G. Festas, P. Kitrou, L. Reppas, K. Palialexis, C. Papageorgiou. Manuscript editing: K. Katsanos, S. Spiliopoulos, P. Kitrou, L. Reppas, K. Palialexis, D. Karnabatidis, I. Paraskevopoulos, M. Mariappan, G. Festas, C. Papageorgiou. All authors have the submitted version. The author(s) read and approved the final manuscript.

\section{Funding}

This research did not receive any specific grant from funding agencies in the public, commercial, or not-for-profit sectors.

\section{Availability of data and materials}

The datasets used and/or analysed during the current study are available from the corresponding author on reasonable request.

\section{Ethics approval and consent to participate}

Institutional review board approval was not required for this retrospective study. A specific study informed consent was not necessary for inclusion in this retrospective study. A procedural written consent form has been obtained by all patients.

\section{Consent for publication}

Not applicable.

\section{Competing interests}

The authors declare that they have no competing interests.

\section{Author details}

${ }^{1}$ 2nd Department of Radiology, Interventional Radiology Unit, Medical School, National and Kapodistrian University of Athens, "Attikon" University Hospital, Athens, Greece. ${ }^{2}$ Department of Clinical Radiology, Interventional Radiology Unit, Aberdeen Royal Infirmary, NHS Grampian, Aberdeen AB25 2ZN, UK. ${ }^{3}$ Department of Interventional Radiology, School of Medicine, Patras University Hospital, Rion, Greece.

Received: 15 September 2020 Accepted: 29 November 2020 Published online: 10 December 2020

\section{References}

Aina R, Oliva VL, Therasse E et al (2001) Arterial embolotherapy for upper gastrointestinal hemorrhage: outcome assessment. J Vasc Interv Radiol 12: 195-200

Angle JF et al (2010) Quality improvement guidelines for percutaneous transcatheter embolization Society of Interventional Radiology Standards of Practice Committee. J Vasc Interv Radiol 21(10):1479-1486
Baron TH, Kamath PS, McBane RD (2013) Management of antithrombotic therapy in patients undergoing invasive procedures. N Engl J Med 368(22):2113-2124

Bauer JR et al (2004) Transcatheter arterial embolization in the trauma patient: a review. Semin Intervent Radiol 21(1):11-22

Bookstein JJ, Goldstein HM (1973) Successful management of postbiopsy arteriovenous fistula with selective arterial embolization. Radiology. 109(3): 535-536

Chakraverty S, Flood K, Kessel D et al (2012) CIRSE quidelines: quality improvement guidelines for endovascular treatment of traumatic hemorrhage. Cardiovasc Intervent Radiol 35(3):472-482

Chun JY, Morgan R, Belli AM (2010) Radiological management of hemoptysis: a comprehensive review of diagnostic imaging and bronchial arterial embolization. Cardiovasc Intervent Radiol 33:240-250

Coccolini F, Stahel PF, Montori G et al (2017) Pelvic trauma: WSES classification and guidelines. World J Emerg Surg 12:5

Davidson JC, Rahim S, Hanks SE et al (2019) Society of Interventional Radiology Consensus Guidelines for the Periprocedural Management of Thrombotic and Bleeding Risk in patients undergoing percutaneous image-guided interventions-part l: review of anticoagulation agents and clinical considerations: endorsed by the Canadian Association for Interventional Radiology and the Cardiovascular and Interventional Radiological Society of Europe. J Vasc Interv Radiol 30(8):1155-1167

Filippiadis DK, Binkert C, Pellerin O, Hoffmann RT, Krajina A (2017) Pereira PL cirse quality assurance document and standards for classification of complications: the cirse classification system. Cardiovasc Intervent Radiol 40(8):1141-1146

Hur S et al (2014) Safety and efficacy of transcatheter arterial embolization for lower gastrointestinal bleeding: a single-center experience with 112 patients. J Vasc Interv Radiol 25(1):10-19

Jaffe TA, Raiff D, Ho LM, Kim CY (2015) Management of anticoagulant and antiplatelet medications in adults undergoing percutaneous interventions. AJR Am J Roentgenol 205:421-428

Loffroy R, Rao P, Ota S et al (2010) Embolization of acute nonvariceal upper gastrointestinal hemorrhage resistant to endoscopic treatment: results and predictors of recurrent bleeding. Cardiovasc Intervent Radiol 33:1088-1100

Lopera JE (2010) Embolization in trauma: principles and techniques. Semin Intervent Radiol 27(1):14-28

Maegele M (2019) The diagnosis and treatment of acute traumatic bleeding and coagulopathy. Dtsch Arztebl Int 116(47):799-806

Melloul E, Denys A, Demartines N (2015) Management of severe blunt hepatic injury in the era of computed tomography and transarterial embolization: a systematic review and critical appraisal of the literature. J Trauma Acute Care Surg 79(3):468-474

Né R, Chevallier O, Falvo N et al (2018) Embolization with ethylene vinyl alcohol copolymer $\left(O n y x^{\oplus}\right)$ for peripheral hemostatic and non-hemostatic applications: a feasibility and safety study. Quant Imaging Med Surg 8(3):280290

Padia SA, Ingraham CR, Moriarty JM et al (2020) Society of Interventional Radiology Position Statement on Endovascular Intervention for Trauma. J Vasc Interv Radiol 31(3):363-369.e2

Patel IJ, Davidson JC, Nikolic B et al (2012) Consensus guidelines for periprocedural management of coagulation status and hemostasis risk in percutaneous image-guided interventions. J Vasc Interv Radiol 23(6):727736

Pearl IJ et al (2019) Society of Interventional Radiology Consensus Guidelines for the Periprocedural Management of Thrombotic and Bleeding Risk in patients undergoing percutaneous image-guided interventions-part II: recommendations. J Vasc Interv Radiol 30:1168-1184

Ramaswamy RS et al (2014) Role of interventional radiology in the management of acute gastrointestinal bleeding. World J Radiol 6(4):82-92

Revel-Mouroz P, Mokrane FZ, Collot S et al (2015) Hemostastic embolization in oncology. Diagn Interv Imaging 96(7-8):807-821

Rösch J, Dotter CT, Brown MJ (1972) Selective arterial embolization. A new method for control of acute gastrointestinal bleeding. Radiology. 102(2):303306

Shi ZX, Yang J, Liang HW, Cai ZH, Bai B (2017) Emergency transcatheter arterial embolization for massive gastrointestinal arterial hemorrhage. Medicine (Baltimore) 96(52):e9437

Spiliopoulos S, Festas G, Theodosis A et al (2019) Incidence and endovascular treatment of severe spontaneous non-cerebral bleeding: a single-institution experience. Eur Radiol 29(6):3296-3307 
Spiliopoulos S, Inchingolo R, Lucatelli P et al (2018) Transcatheter arterial embolization for bleeding peptic ulcers: a multicenter study. Cardiovasc Intervent Radiol 41(9):1333-1339

Toyoda H, Nakano S, Kumada T et al (1996) Estimation of usefulness of N-butyl-2cyanoacrylate-lipiodol mixture in transcatheter arterial embolization for urgent control of life-threatening massive bleeding from gastric or duodenal ulcer. J Gastroenterol Hepatol 11:252-258

Valek V, Husty J (2013) Quality improvement guidelines for transcatheter embolization for acute gastrointestinal nonvariceal hemorrhage. Cardiovasc Intervent Radiol 36:608-612

\section{Publisher's Note}

Springer Nature remains neutral with regard to jurisdictional claims in published maps and institutional affiliations.

\section{Submit your manuscript to a SpringerOpen ${ }^{\circ}$ journal and benefit from:}

- Convenient online submission

- Rigorous peer review

- Open access: articles freely available online

- High visibility within the field

- Retaining the copyright to your article

Submit your next manuscript at $\boldsymbol{\nabla}$ springeropen.com 